bDMARDs for COVID-risk may cause exacerbation of the primary rheumatic disease, continuing with current treatments seems an appropriate approach. REFERENCES:

[1] FELDSTEIN LR, ROSE EB, HORWITZ SM, et al: Overcoming COVID-19 Investigators; CDC COVID-19 Response Team. Multisystem Inflammatory Syndrome in U.S. Children and Adolescents. N Engl J Med 2020; 383: 334-46.

[2] MICHELENA X, BORRELL H, LÓPEZ-CORBETO $M$, et al. Incidence of COVID-19 in a cohort of adult and paediatric patients with rheumatic diseases treated with targeted biologic and synthetic disease-modifying anti-rheumatic drugs. Semin Arthritis Rheum 2020; 50: 564-70.

Disclosure of Interests: None declared.

DOI: 10.1136/annrheumdis-2021-eular.1173

\section{POS1182 MANAGEMENT OF DIFFICULT-TO-TREAT RHEUMATOID ARTHRITIS DURING THE COVID- 19 PANDEMIC: EVIDENCE FROM THE ITALIAN EPICENTER}

F. Ingegnoli ${ }^{1}$, A. F. Luppino ${ }^{1}$, G. Cincinelli ${ }^{1}$, E. Favalli ${ }^{1}$, R. Caporali ${ }^{1}{ }^{1}$ Università degli Studi di Milano, Division of Clinical Rheumatology, ASST Pini, Department of Clinical Sciences \& Community Health, Research Center for Adult and Pediatric Rheumatic Diseases, Milano, Italy

Background: Despite significant improvement in the RA management, up to twenty percent of patients with rheumatoid arthritis (RA) have a difficult-to-treat (D2T) disease. The COVID-19 related mitigation policies, for instance quarantine, and consequent difficult access to in-person visits, laboratory and imaging investigations, adversely affected the follow up of rheumatic patients. Although pandemic-imposed limitations could have negatively influenced disease management particularly in D2T patients, to what degree these restrictions affected the treat-to target (T2T) and tight-control strategy in this subgroup of RA patients has not been investigated yet.

Objectives: To evaluate whether the switch to telehealth imposed by COVID19 pandemic was effective in the management of D2T RA patients treated with targeted therapies.

Methods: This observational retrospective real-life study was conducted from November 2019 through September 2020. Among RA patients treated with targeted therapies, RA D2T patients according to EULAR definition (1) were identified. Clinical Disease Activity Index (CDAI) of these patients was analysed retrospectively before, during and after lockdown (LD). During LD period, patients could choose whether to receive home drug delivery or to maintain their face-to-face consultations, and in the former rheumatologists provided virtual care. To evaluate the effect of LD on the percentage of patients in remission, logistic mixed effects regression models were fitted, with CDAI remission as response variable.

Results: Data were extracted from a longitudinal observational registry, and at baseline, 52 patients treated with targeted therapies were classified as D2T RA. Among them, during pre-LD, LD, and post-LD $11.54 \%(\mathrm{~N}=6), 53.49 \%$ $(\mathrm{N}=23)$, and $46.15 \%(\mathrm{~N}=24)$ had $\mathrm{CDAl}$ remission/low disease activity, while $46(88.46 \%), 20(46.51 \%)$ and $28(53.85 \%)$ had CDAl moderate/high. All the patients completed the follow-up. Median values of CDAl during pre-LD, LD, and post-LD were 14.5 [IQR 12-21], 9 [IQR 5.5-16], and 11 [IQR 6-19.2] respectively (see Figure 1 below).

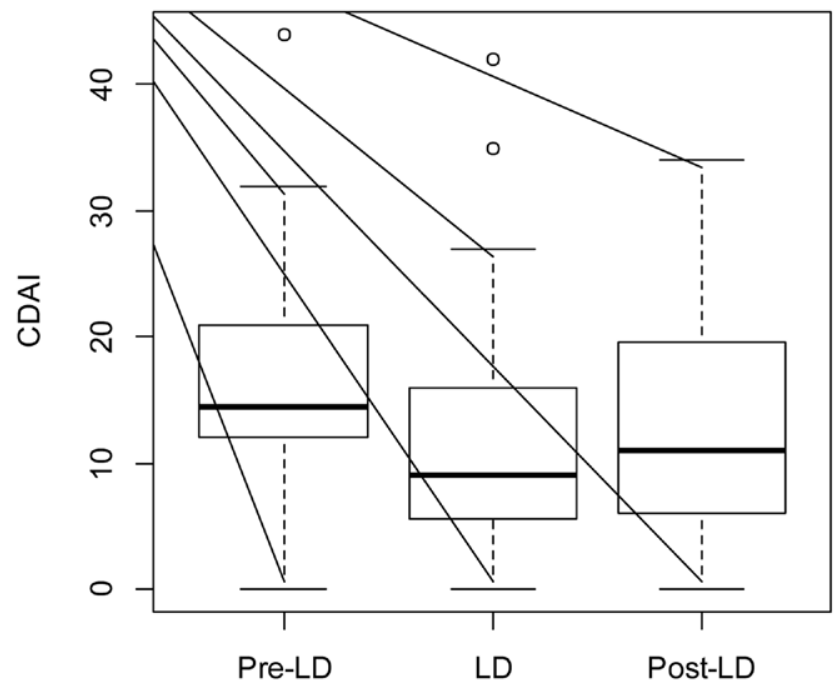

Conclusion: Telephone-based tight control strategy ensured satisfactory management of D2T RA treated with targeted therapies. This temporary approach has been a feasible compensation for the decline of face-to-face visits also in this challenging group of RA patients, thus reassuring for future months before the end of pandemic.

\section{REFERENCES:}

[1] Nagy G, et al. EULAR definition of difficult-to-treat rheumatoid arthritis. Ann Rheum Dis 2021;80(1):31-35.

Disclosure of Interests: Francesca Ingegnoli: None declared, Angela Flavia Luppino: None declared, Gilberto Cincinelli: None declared, Ennio Favalli Speakers bureau: AbbVie, Sanofi-Genzyme, Lilly, UCB, Pfizer, Novartis, Janssen, Paid instructor for: Roche, MSD, Consultant of: Lilly, Galapagos, Roberto Caporal Speakers bureau: Abbvie, Amgen, BMS, Celltrion, Galapagos, Gilead, Lilly, Pfizer, Roche, UCB, Sanofi, Fresenius Kabi, Samsung bioepis, MSD, Consultant of: Galapagos, Gilead, Lilly,Janssen, MSD. DOI: 10.1136/annrheumdis-2021-eular.1188

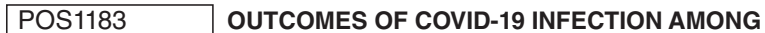 CHILDREN AND YOUNG PEOPLE WITH PRE-EXISTING RHEUMATIC AND MUSCULOSKELETAL DISEASES}

L. Kearsley-Fleet ${ }^{1}$, S. Lawson-Tovey ${ }^{2,3}$, R. E. Costello ${ }^{1}$, A. Belot ${ }^{4}$

F. Aeschlimann ${ }^{5,6}$, I. Melki ${ }^{5,6,7}$, I. Koné-Paut ${ }^{8}$, D. Clemente ${ }^{9}$, M. C. Pinedo Gago $^{10}$, N. Svestkova ${ }^{11}$, N. Vinšová ${ }^{11}$, M. Hamad Saied ${ }^{12}$, Y. Berkun ${ }^{13}$, N. Wulffraat $^{14}$, S. Eulert ${ }^{15}$, C. A. Scirè ${ }^{16}$, A. Strangfeld ${ }^{15}$, E. Mateus ${ }^{17,18}$, P. Machado ${ }^{19,20,21}$, Y. Uziel ${ }^{22,23}$, K. Hyrich ${ }^{1,3}$. $^{1}$ The University of Manchester, Centre for Epidemiology Versus Arthritis, Manchester, United Kingdom; ${ }^{2}$ The University of Manchester, Centre for Genetics and Genomics Versus Arthritis, Centre for Musculoskeletal Research, Manchester, United Kingdom; ${ }^{3}$ Manchester University NHS Foundation Trust, Manchester Academic Health Science Centre, National Institute of Health Research Manchester Biomedical Research Centre, Manchester, United Kingdom; ${ }^{4}$ Hospices Civils de Lyon, Pediatric Rheumatology Unit, Lyon, France; ${ }^{5}$ Hôpital Necker-Enfants Malades, Pediatric Hematology-Immunology and Rheumatology Department, Reference center for Rheumatic, Autolmmune and Systemic diseases in children (RAISE) \& FAI2R, Paris, France; ${ }^{6}$ Paris University, Laboratory of Immunogenetics of Paediatric Autoimmunity, Imagine Institute, Paris, France; ${ }^{7}$ Hôpital Robert Debre, General Pediatrics, Infectious Disease and Internal Medicine Department, Reference center for Rheumatic, Autolmmune and Systemic diseases in children (RAISE) \& FAI2R, Paris, France; ${ }^{8}$ University of Paris Sud Saclay, CeRéMAIA, FAI2R, AP-HP, Bicêtre Hospital, Paris, France; ${ }^{9}$ Hospital Niño Jesús, Unidad de Reumatología Pediátrica, Madrid, Spain; ${ }^{10} \mathrm{Hospital}$ Universitario Cruces, Reumatología Pediátrica, Barakaldo, Spain; ${ }^{11}$ Charles University and General University Hospital, Centre for Paediatric Rheumatology and Autoinflammatory Diseases, Department of Paediatrics and Inherited Metabolic Disorders, 1st Faculty of Medicine, Prague, Czech Republic;

${ }^{12}$ Carmel Medical Centre, Pediatrics Rheumatology Unit, Haifa, Israel; ${ }^{13} \mathrm{Hebrew}$ University of Jerusalem, Department of Pediatrics, Hadassah-Hebrew University Medical Center, Mount Scopus, Faculty of Medicine, Jerusalem, Israel; ${ }^{14}$ University Medical Center, Department of Pediatric Immunology and Rheumatology, Wilhelmina Children's Hospital, Utrecht, Netherlands; ${ }^{15}$ German Rheumatism Research Center (DRFZ Berlin), Epidemiology and Health Care Research Unit, Berlin, Germany; ${ }^{16}$ University of Ferrara, Rheumatology Unit, Department of Medical Sciences, Ferrara, Italy; ${ }^{17}$ Portuguese League Against Rheumatic Diseases, LPCDR, Lisbon, Portugal; ${ }^{18}$ European League Against Rheumatism (EULAR) Standing Committee, People with Arthritis/Rheumatism in Europe (PARE), Kilchberg, Switzerland; ${ }^{19}$ University College London, Centre for Rheumatology \& Department of Neuromuscular Diseases, London United Kingdom; ${ }^{20}$ University College London Hospitals National Health Service (NHS) Trust, National Institute for Health Research (NIHR) University College London Hospitals Biomedical Research Centre, London, United Kingdom; ${ }^{21}$ London North West University Healthcare NHS Trust, Department of Rheumatology, Northwick Park Hospital, London, United Kingdom; ${ }^{22}$ Meir Medical Center, Pediatric Rheumatology Unit, Department of Pediatrics, KfarSaba, Israel; ${ }^{23}$ Tel Aviv University, Sackler Faculty of Medicine, Tel Aviv, Israel

Background: It remains unknown whether children and young people with rheumatic and musculoskeletal diseases (RMD) who acquire COVID-19 infection have a more severe COVID-19 course, due to either underlying disease or immunosuppressive treatments.

Objectives: To describe outcomes among children and young people with underlying RMD who acquire COVID-19 infection.

Methods: All children and young people $<18$ years of age with COVID-19 (presumptive or confirmed) reported to the EULAR COVID-19 Database, which collects details regarding RMD diagnosis and treatment, COVID infection and outcomes, between 27 March 2020 and 29 January 2021 (cutoff date for this analysis) were included. Patient characteristics and COVID-19 outcomes are presented. 